\title{
A developmentally regulated trans-acting factor recognizes dissimilar G/C-rich elements controlling a class of cAMP- inducible Dictyostelium genes
}

\author{
Annegrethe L. Hjorth, ${ }^{1,4}$ Catherine Pears, ${ }^{2,5}{ }^{\text {Jeffrey G. Williams, }}{ }^{2}$ and Richard A. Firtel ${ }^{1,3}$ \\ IDepartment of Biology, Center for Molecular Genetics M-034, University of California, San Diego, La Jolla, California 92093 \\ USA; ${ }^{2}$ Imperial Cancer Research Fund, Clare Hall Laboratories, Herts EN6 3LD UK
}

\begin{abstract}
Transcriptional response elements involved in the cAMP-inducible and developmentally regulated expression of the Dictyostelium aggregate-stage gene pst-cath/CP2 have been shown to include a G/C-rich sequence element [G-box regulatory element (GBRE)]. We have recently identified a trans-acting factor, GBF (GBRE binding factor), that specifically interacts with this sequence and have shown that the binding activity of GBF to GBRE is developmentally regulated and inducible by cAMP. Here, we examine further the possible role of GBF in the regulation of $p s t$-cath/CP2 and three other coordinately regulated, cAMP-inducible aggregate-stage genes. We show that GBF itself (or other closely related factors) recognizes dissimilar G/C-rich elements present in the 5'-flanking regions of these genes and that the ability of the individual, distinct G/C-rich elements to confer regulated expression on a promoter deletion mutant of the pst-cath/CP2 gene is correlated with the relative affinity for GBF. G/C-rich elements carrying point mutations that prevent in vitro binding of GBF to two of the G/C-rich elements fail to activate expression in vivo. An analysis of major points of contact between the GBF protein and two distinctly different binding sites suggests that binding of GBF to these sequence elements involves a considerable degree of flexibility in DNA-protein interactions. These results suggest that the regulated expression of a class of aggregate-stage cAMP-inducible genes involves the interaction of GBF or homologous factors with dissimilar G/C-rich sequence elements and that induction of GBF activity or that of homologous factors by cAMP may thus be a limiting step in the induction of this temporally coordinate set of genes during Dictyostelium development.
\end{abstract}

[Key Words: Dictyostelium; cAMP; gene expression]

Received October 3, 1989; revised version accepted December 20, 1989.

Dictyostelium discoideum propagates as a vegetative amoeba in the presence of nutrients and initiates, upon starvation, a multicellular differentiation process that results in the formation of a mature fruiting body containing two distinctly different cell types-stalk cells and spores. Within several hours of starvation, Dictyostelium cells aggregate in response to pulsatile cAMP signals. Binding of cAMP to cell-surface receptors activates adenylate cyclase, and repeated cycles of cAMP synthesis and secretion serve to transmit, or relay, the chemotactic signal outward through the population of aggregating cells (see Janssens and van Haastert 1987; Firtel et al. 1989). After formation of tightly associated multicellular aggregates, a migrating pseudoplasmodium (slug) is formed in which precursor cells to the

${ }^{3}$ Corresponding author.

Present addresses: ${ }^{4}$ Novo-Nordisk A/S, Novo Allé, DK-2880 Bagsvaerd, Denmark; ${ }^{5}$ Ludwig Institute, London W1P 8T UK. fully differentiated stalk and spore cells (prestalk and prespore cells) are spatially separated. The prestalk cells are localized in the anterior part of the pseudoplasmodium, whereas the posterior $\sim 80 \%$ of the slug is composed of prespore cells (Loomis 1975, 1982).

Prespore genes (preferentially expressed in prespore cells) and another class, which show preferential expression in the anterior cells of the slug and have thus been designated prestalk genes, are induced at the time of multicellular aggregate formation (Barklis and Lodish 1983; Mehdy et al. 1983; Reymond et al. 1984; Pears et al. 1985; Datta et al. 1986; Gomer et al. 1986a; Jermyn et al. 1987). The expression of both classes of aggregatestage genes in single-cell, developmental cultures is induced by high levels of cAMP and is regulated through activation of cell-surface cAMP receptors through an intracellular signal transduction pathway that does not appear to require a rise in intracellular CAMP or the activation of cAMP-dependent protein kinase A /Schaap and van Driel 1985; Gomer et al. 1986b; Haribabu and 
Dottin 1986; Oyama and Blumberg 1986; Kimmel 1987; Schaap et al. 1987; Mann et al. 1989; Firtel and Chapman 1990). In addition, a class of prestalk-specific, aggregate-stage genes is induced by the morphogen DIF at approximately the same time in development (Williams et al. 1987).

Promoter analysis of the cAMP-induced, prestalkenriched gene pst-cathepsin (Datta and Firtel 1987), or CP2 (Pears and Williams 1987; henceforth called pstcath/CP2), identified an essential 80-bp cis-acting regulatory region that contains a G-rich element, designated GBRE (G-box regulatory element), the deletion of which results in a 50- to 100-fold reduction in the level of expression. Other regulatory elements, in addition to GBRE, were also identified and shown to affect the level of expression and, in some instances, the relative response to developmental or cAMP signals. The residual expression found in the GBRE deletions is still developmentally regulated, suggesting that other elements of the promoter respond to developmental signals (Datta and Firtel 1987, 1988; Pears and Williams 1987). Insertion of oligonucleotides containing the wild-type GBRE restores high levels of regulated expression, whereas a series of mutated GBRE sequences do not (Hjorth et al. 1989; this paper).

A nuclear factor called GBF (GBRE-binding factor) has been identified that specifically interacts with the GBRE of pst-cath/CP2 promoter (Hjorth et al. 1989). The level of GBRE binding is developmentally induced 20- to 50fold with kinetics that correspond to the developmental kinetics of pst-cath/CP2 expression. GBRE-binding activity is also inducible by cAMP in shaking cultures, and this increase is sensitive to cycloheximide, suggesting that either the factor itself or a protein required for the increase in GBF activity is synthesized de novo in response to cAMP. Point mutations within the GBRE that eliminate complementation of the pst-cath/CP2 promoter in vivo also do not compete for binding of the factor in vitro to the pst-cath/CP2 GBRE. These point mutations affect specific $G$ residues that are required for binding as determined by dimethylsulfate (DMS) methylation interference studies. These results led to the proposition that GBF mediates GBRE activation of pstcath/CP2 gene expression in vivo.

In this paper we have extended the analysis of GBF and examined its interaction with G/C-rich sequence elements from four cAMP-induced, aggregate-stage genes, all of which are transcriptionally induced by cAMP, suggesting a common regulatory mechanism for the genes. In addition to pst-cath/CP2, we have analyzed three other genes: another cysteine protease, CP1; the DG17 gene, which is tightly linked to and coordinately regulated with CP1 (Williams et al. 1985; Driscoll and Williams 1987); and the gene encoding UDP glucose pyrophosphorylase (UDPGPP). The UDPGPP gene is expressed in vegetative cells in early development and, at an increased level, during multicellular stages (Haribabu et al. 1986, 1988; Ragheb and Dottin 1986; Pavlovic et al. 1988). All three of these genes have a G/C-rich element and, for CP1, this element has been shown to confer cAMP-induced expression on a pst-cath/CP2 promoter construct carrying a deletion of the GBRE (Pears and Williams 1988). In this paper, we describe the relative ability of wild-type and mutant oligonucleotides containing these different G/C-rich elements to complement the pst-cath/CP2 GBRE deletion mutant and to interact with GBF in vitro. From the results presented here, we suggest that GBF or a set of related factors may interact with diverse G/C-rich sequence elements from all of these genes and that GBF may be a common transacting factor involved in regulating the expression of this class of aggregate-stage, cAMP-inducible genes in vivo.

\section{Results}

\section{Structure of the G/C-rich elements}

The structural organization of the upstream regions of the four aggregate-stage, cAMP-inducible Dictyostelium genes is presented in Figure 1, with an emphasis on known and putative G/C-rich cis-acting regulatory elements. Although all of these genes have been shown to be transcriptionally induced by cAMP and show similar developmental kinetics of expression, the only apparent similarity between the putative regulatory elements is that they are G/C rich (Williams et al. 1980; Mehdy et al. 1983; Mehdy and Firtel 1985; Pears et al. 1985; Williams et al. 1985; Datta et al. 1986; Haribabu et al. 1986; Driscoll and Williams 1987; Pavlovic et al. 1989). The rest of the $5^{\prime}$-flanking regions are $>90 \% \mathrm{~A} / \mathrm{T}$, similar to the majority of the $5^{\prime}$-flanking regions of other Dictyostelium genes (Kimmel and Firtel 1983).

The pst-cath/CP2 GBRE, the best characterized of these elements, consists of $5^{\prime}$ and $3^{\prime}$ domains that have two nucleotide sequence differences within the G-rich core. A full level of expression requires the presence of both domains at an appropriate spacing (Datta and Firtel 1987, 1988; Pears and Williams 1987). Insertion of oligonucleotides, including both the G-box domains, into a pst-cath/CP2 promoter deletion restores cAMP inducibility and substantially stimulates expression of the transformed gene in multicellular aggregates (Hjorth et al. 1989).

Previous results also show that insertion of a long oligonucleotide that includes a 60 -bp CP1 5' element containing two related short G-rich elements into a pstcath/CP2 promoter deletion restored a high level of cAMP-inducible expression (Pears and Williams 1988). Insertion of a single CP1 G-rich element had a very small stimulatory effect, whereas insertion of two copies of this oligonucleotide resulted in a significant enhancement of expression, strongly suggesting that the minimum CP1 element consists of two G-rich regions and that this element serves a function homologous to that of the two domains of the pst-cath/CP2 GBRE. Initial deletion analysis of the UDPGPP regulatory region showed that essential cis-acting elements needed for cAMP induction are located in the region that includes the palindromic UDPGPP G/C-rich sequence (Fig. 1), 

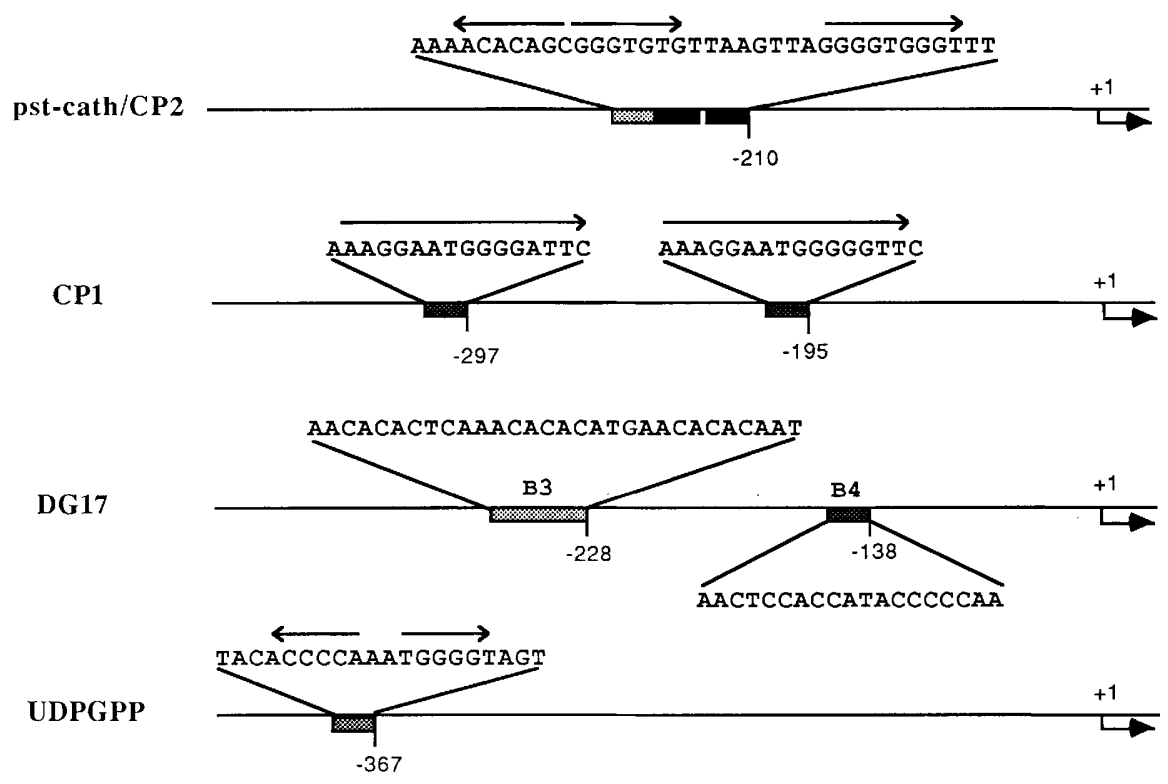

pst-cath/CP2: w $t$ ATTTATGAAAACACAGCGGGTGTGTAAGTTAGGGGTGGGTITATATA

$\triangle 30 \quad$ ATTTATGAAAACACggatccgTATATA

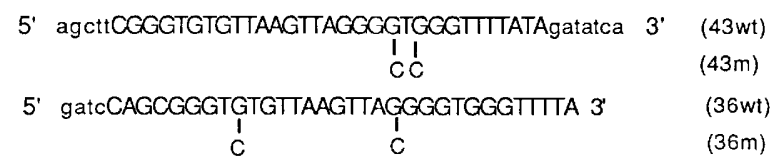

CP1:

$$
\begin{array}{ll}
\text { DG17-B3: } & 5^{\prime} \text { gatcTACAAACACACTCAAACACACATGAACACACAg } 3^{\prime} \\
\text { DG17-B4: } & 5^{\prime} \text { gatcTACCACATAACAACTCCACCATACCCOCAAg } 3^{\prime} \\
\text { UDPGPP: } & 5^{\prime} \text { gatccTITAACTACACCCCAAATGGGGTAGTTGAAA } 3
\end{array}
$$

Figure 1. Organization of G/C-rich elements in upstream regions of aggregate-stage cAMP-inducible genes. (Top) The core of the G/C-rich sequence elements discussed in the text is presented, and the positions of the elements relative to the Cap site are indicated. The pattern of the boxes, representing the G/C-rich elements, emphasizes sequence homologies between the various elements. Direct or inverted repeat structures within a given element are indicated by arrows above the G/C-rich sequences. The two G/C-rich elements present in the upstream region of the DG17 gene are referred to as B3 (Box3) and B4 (Box4) in agreement with previously published notation (Driscoll and Williams 1987). (Bottom) Sequences of wild-type and mutant oligonucleotides used in this analysis. Uppercase letters refer to sequences derived from the Dictyostelium genome; lowercase letters represent additional sequences that provide the oligonucleotides with GATC sticky ends, with the exception of the pst-cath/CP2 43-mer oligonucleotides that have HindIII $5^{\prime}$ overhangs at the ends. The specific changes, introduced to create the mutant versions of the pst-cath/CP2 and CP1 oligonucleotides, are indicated below the corresponding wild-type sequence. The two CP1 G-rich elements shown are each part of an $\sim 60$-bp repeated element. Each of these two 60 -bp elements contains two copies of the respective $C P 1 \mathrm{G}$-rich sequences shown (for references, see text $\}$. The sequence of $C P 2 \Delta 30(\triangle 30)$ around the site of deletion of the GBRE is also shown. The lowercase letters represent the BamHI linker sequence (for references, see text).

which corresponds to the site of an inducible DNase I hypersensitive site (Ragheb and Dottin 1986; Haribabu et al. 1988; Pavlovic et al. 1988). Recent data definitely show that this element is required for cAMP-induced expression (Pavlovic et al. 1989).
The two sequence elements (DG17-B3 and -B4), which are distinguished by being exceptionally G/C-rich in an otherwise very A/T-rich region, are located at the approximate position of DNase I hypersensitive sites that become induced during development when the DG17 
gene is first expressed (Pavlovic et al. 1986; Driscoll and Williams 1987). Of these two elements, the gene-proximal one (B4) shows a striking resemblance to an inverted copy of the CP1 G-rich element, whereas the $D G 17-\mathrm{B} 3$ element bears no apparent sequence homology to either the pst-cath/CP2 or the CP1 G-rich elements.

\section{In vitro binding of $G / C$ elements to nuclear factors}

We have analyzed DNA-protein complex formation in vitro with the various G/C-rich elements, using a partially purified nuclear extract prepared from 15 -hr aggregates. Binding was performed using fragments that contained either a single or a tandem duplication of the oligonucleotides presented in Figure 1 and analyzed by gel retardation. The intensity of the low-mobility bands seen in Figure 2A (the DNA-protein complexes) reflects the relative affinity of nuclear factor(s) present in our extract for these sequences. Note that one copy of the $C P 1$ sequence binds very poorly (a very low level of binding is detected), whereas two copies bind very well. DG17-B4 and UDPGPP show poor binding with one copy and very weak binding with two copies when compared to the binding with two copies of the other three probes.

In a previous analysis of the specificity of GBF for the pst-cath/CP2 GBRE, we demonstrated that introduction of specific point mutations into the GBRE (mutants $43 \mathrm{~m}$ and $36 \mathrm{~m}$ in Fig. 1) reduces the affinity of the GBRE for GBF at least 50-fold and, moreover, that these mutant oligonucleotides do not restore an elevated pst-cath/CP2 expression in vivo when they are inserted into a pstcath/CP2 GBRE deletion construct (CP2 30 ) (Hjorth et al. 1989; see also Fig. 5, below). To examine whether the protein factor(s) that binds the CP1, UDPGPP, and $D G 17 \mathrm{G} / \mathrm{C}$-rich elements might be identical or related to the one(s) that interacts with the pst-cath/CP2 GBRE, we performed a gel shift competition analysis using wild-type and mutant pst-cath/CP2 GBRE oligonucleotides as competitors (see legend to Fig. 2B). As seen in Figure 2B, a 50-fold molar excess of the two wild-type pst-cath/CP2 oligonucleotides (labeled $43 \mathrm{w}$ and $36 \mathrm{w}$ ) competed very well for the binding to each of the probes, whereas neither of the mutant oligonucleotides $143 \mathrm{~m}$ and $36 \mathrm{~m}$ ) showed significant competition (for sequences, see Fig. 1). These results suggest that the same protein/s), or members of a family of related factors, forms complexes with each of the G/C-rich elements, including the pst-cath/CP2 GBRE.

A quantitative comparison of the ability of each of the G/C-rich oligonucleotides to compete for binding of nuclear factors to the pst-cath/CP2 GBRE was obtained in a separate competition analysis. In these experiments, the binding to one or two copies of the pst-cath/CP2 oligonucleotides was competed by the addition of varying amounts of unlabeled G/C-rich DNA fragments. Competition curves resulting from a densitometric analysis of such experiments are shown in Figure 3. The relative order of binding affinity observed using cloned fragments carrying one copy of each oligonucleotide as a competitor and a one-copy pst-cath/CP2-binding sub- strate are $D G 17-\mathrm{B} 3>C P 2>D G 17-\mathrm{B} 4 \sim U D P G P P>$ $C P 1$. A similar relative order of competition is preserved for the two-copy clones, with the exception that two copies of CP1 compete fairly well. As shown in Figure 2, $C P 1$ shows barely detectable binding as a single copy, yet is a medium-strength binding site as a duplicated sequence, consistent with the competition studies shown here. These results agree with the relative ability of each of the G/C-rich fragments to form protein-DNA complexes seen in Figure 2 and is consistent with the possibility that GBF or a related family of proteins binds to each of the sequences (see Discussion).

To better evaluate the results from this competition analysis, we have examined the binding parameters of the standard assay in more detail. A titration analysis, using one and two copies of the pst-cath/CP2 element as probe and increasing amounts of extract, shows a saturation effect that is reached at $\sim 5 \%$ binding of probe for the one-copy substrate and at $\sim 10 \%$ for two copies (see Fig. 4). We assume that the inability to obtain quantitative retention of the DNA with this extract preparation is caused by several factors, including the relative binding affinities of GBF for GBRE and "nonspecific" competitor DNAs and the presence of nonspecific, competing DNA-binding proteins that are not competed completely with poly[d(I-C)] or a variety of other DNAs tested. Such a curve helps explain the nonstoichiometric competition analysis seen in Figure 3.

\section{G-rich oligonucleotide-directed expression in vivo}

The above results suggest that the G/C-rich elements from the four genes bind to GBF, or a related set of factors with substantial differences in the relative binding affinities. To assess the possible function of the G/C-rich sequence elements in vivo, we have inserted the elements as oligonucleotides into the pst-cath/CP2 minigene promoter construct $(C P 2 \Delta 30)$ (Pears and Williams 1987, 1988; Hjorth et al. 1989) (see Fig. 1) and examined expression during multicellular development and in cAMP-induced, single-cell culture. The CP2A30 $(\Delta 30)$ construct carries a 30 -bp deletion in the $5^{\prime}$ flanking sequence that removes the entire GBRE and inserts a BamHI site and a deletion within the proteincoding region, which results in a minigene transcript that can be separated easily from the endogenous gene on RNA blots. Expression of the minigene mRNA is 25to 100 -fold lower from this construct compared to that directed by a wild-type promoter during multicellular development. The wild-type $p s t$-cath/CP2 construct is highly responsive to exogenous cAMP in single-cell culture, whereas the $\Delta 30$ minigene is either not detectably expressed (see Fig. 5) or is expressed at a level only $\sim 1 \%$ of the wild-type gene (Pears and Williams 1987). These experimental variations in the level of expression of the CP2 30 construct are probably due to slightly different culturing conditions affecting the relative amount of cellular aggregation formation that occurs in these assays; a higher level of aggregate formation results in a higher level of expression of $\Delta 30$. 


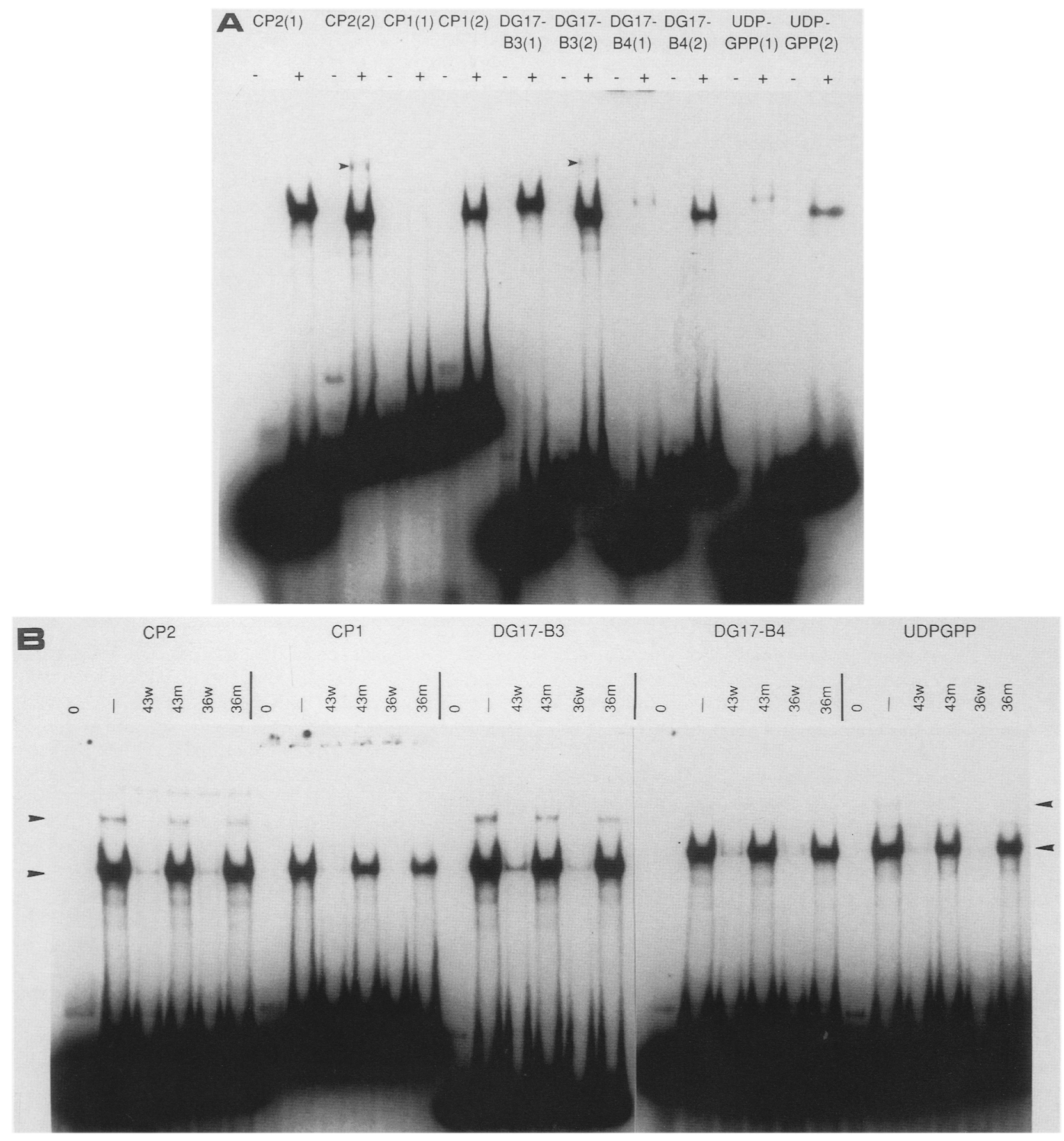

Figure 2. Binding of GBF to G/C-rich sequence elements. (A) Binding of nuclear factor(s) to one and two copies of the G/C-rich oligonucleotides. Complex formation in vitro was analyzed using ${ }^{32}$ P-labeled DNA fragments that contain a single copy (1) or two copies $(2)$ of the G/C-rich oligonucleotide derived from the pst-cath/CP2,CP1,DG17, and UDPGPP upstream regions, as indicated. DNA fragments were incubated with $(+)$ or without $(-)$ nuclear extract prepared from 15-hr developed Dictyostelium cells and analyzed on native acrylamide gels. Arrowheads indicate a secondary, less intense band that is observed only for the two strongest binding sites, CP2 and DG17-B3. The competition behavior of these bands is indistinguishable from that of the lower, more intense band (see also Hjorth et al. 1989; for details, see Materials and methods). (B) Competition analysis of complexes formed with two-copy clones of G/C-rich elements. Mobility-shift assays were performed using DNA fragments containing two cloned copies of the various G/C-rich elements (see Fig. 1). Complex formation with 15-hr nuclear extract was performed in the presence of a 500-fold weight excess of nonspecific competitor poly[d(I-C)] alone $(-)$ or in combination with a 50-fold weight excess of self-ligated pst-cath/CP2 wild-type $(36 \mathrm{wt}, 43 \mathrm{wt})$ and mutant $(36 \mathrm{~m}, 43 \mathrm{~m})$ oligonucleotides (Fig. 1). Note that the two pst-cath/CP2 GBRE wild-type oligonucleotides ( $36 \mathrm{w}$ and $43 \mathrm{w}$ ) have the same GBRE core and only differ in the number of outside nucleotides and the restriction site at the end. Both behave identically with regard to binding to GBF in vitro and to activation in vivo (Hjorth et al. 1989). Both wild-type copies are used in the competitions as controls for the two mutants, one of which is made in the two different wild-type backgrounds. (Lane 0) Labeled probe incubated without nuclear extract. 
Figure 3. Competition analysis of GBF binding to G/C-rich elements. Competition of $\mathrm{G} / \mathrm{C}$-rich oligonucleotides for the binding of GBF to the CP2 G-box element. The probes used in these assays were DNA fragments containing the pstcath/CP2 43-mer cloned in one copy (top) or two copies (bottom) into pUC18. (Top) The binding assay contained 0.2 ng of labeled probe, a 500 -fold weight excess of poly (dI-dC) as a nonspecific competitor, and a molar excess of DNA fragments containing G/C-rich oligonucleotides, as indicated. With the exception of the mutant pst-cath/CP2 43mer oligonucleotide $[C P 2(43 \mathrm{~m})]$, the DNA fragments contained wild-type sequences of the G/C-rich elements (see Fig. 1). The nuclear extract was added last to the assay mixture. The samples were analyzed on native acrylamide gels, and the relative intensity of the bands, which represent the complexed form of DNA, was quantitated by laser densitometry. Results are presented as a percentage of the binding observed in the absence of specific DNA competitor. (Bottom) An analysis similar to the one presented above, using DNA fragments containing two tandemly arranged oligonucleotides as probe (pst-cath/CP2) and as specific competitors. The lower, more intense band (see Fig. 2) was quantitated.

Figure 5 shows an RNA blot analysis of the relative level of oligonucleotide-directed expression of the minigene at the early finger stage $(\sim 15 \mathrm{hr})$ and following cAMP induction in single cells in shaking culture. No detectable expression of the endogenous pst-cath/CP2 gene or of any of the transformed constructs was observed in vegetatively growing cells or in shaken cells in the absence of exogenous cAMP (data not shown). Quantitation of the data for multicellular development and cAMP induction is presented in Table 1. The CP2 30 construct shows $\sim 4 \%$ of wild-type activity in the developmental assay in this set of experiments (lower in experiments described in Fig. 6). Insertion of

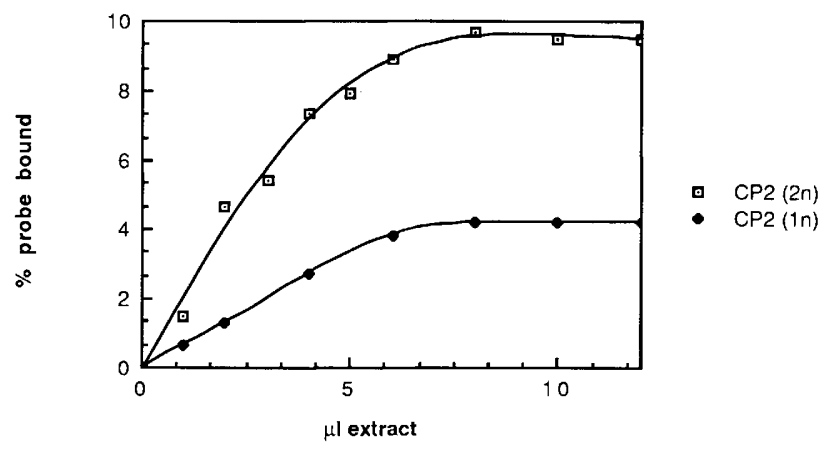

Figure 4. Titration analysis of GBF binding. Increasing amounts of nuclear extract were incubated with ${ }^{32} \mathrm{P}$-labeled DNA fragments containing either one copy (1n) or two copies (2n) of the pst-cath/CP2 43-mer oligonucleotide (Fig. 1). Complex formation was analyzed by electrophoresis on native acrylamide gels and quantitated by laser densitometry of autoradiographs. For the $C P 2(2 n)$ probe, only the more rapidly migrating complex band was quantitated.
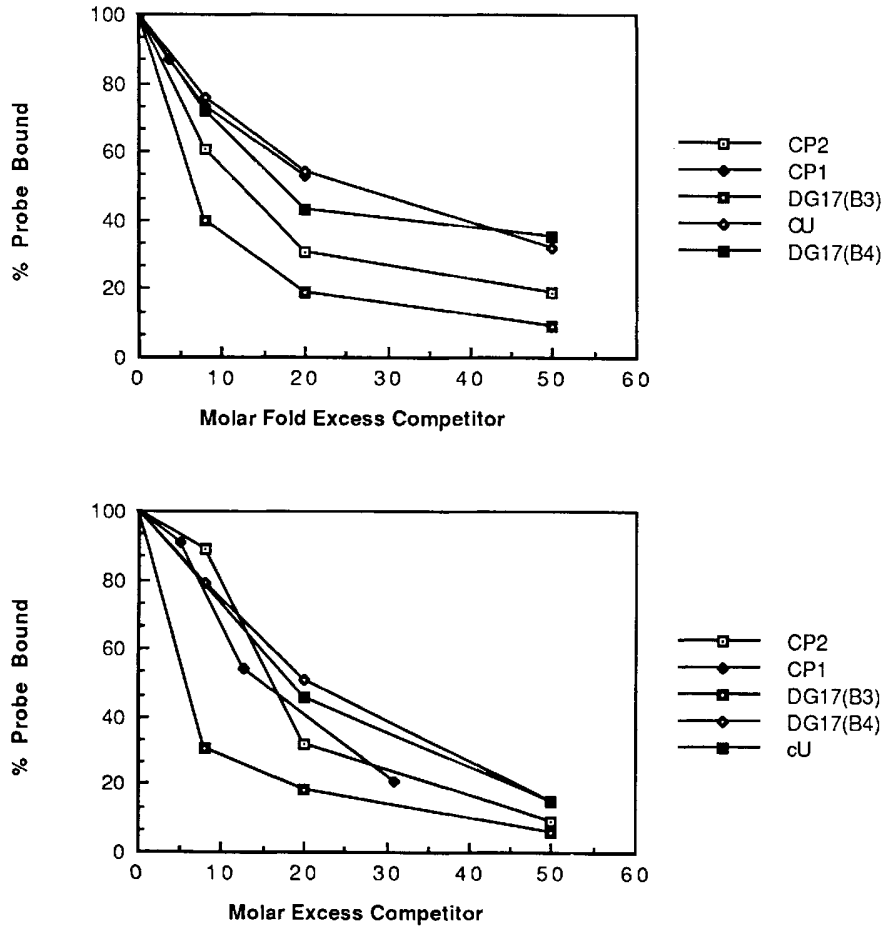

one or two copies (Fig. 5; Hjorth et al. 1989) of a mutant pst-cath/CP2 GBRE oligonucleotide reduces the level of expression to $<1 \%$. Presumably, this is the result of disruption of sequence contacts within the whole pstcath/CP2 regulatory region required for the low level expression of CP2 330 (see Datta and Firtel 1988). Insertion of a single copy of the wild-type pst-cath/CP2 oligonucleotide results in a small stimulation relative to $C P 2 \triangle 30$ but a stimulation $>10$-fold over $C P 2 \Delta 30$ containing a mutant $p s t$-cath/CP2 oligonucleotide. Approximately the same level of stimulation is seen for one copy of DG17-B3, and a negligible stimulation is caused by one copy of the other oligonucleotides. When two copies of DG17-B3, pst-cath/CP2, and CP1 oligonucleotides were inserted as direct repeats, a high level of stimulation was observed, whereas duplicate DG17-B4 and UDPGPP oligonucleotides only conferred a very slight stimulation (see Figs. 5 and 6). Two copies of the pstcath/CP2 mutant oligonucleotide $C P 2(43 \mathrm{~m})$ resulted in $<1 \%$ activity, whereas $C P 2(36 \mathrm{~m})$ had $1 \%$ activity. The two copies of $C P 2, C P 1$, and DG17-B3 thus had $\geqslant 28$-fold stimulation in relation to the $C P 2 \triangle 30 / C P 2(36 \mathrm{~m})$ construct.

Expression of the four genes has been shown to be induced in single-cell shaking culture in response to high, continuous levels of cAMP (see introductory section). This activation is mediated via cell-surface cAMP receptors and does not require a rise in intracellular cAMP (for conditions, see Materials and methods). When cAMP induction of the constructs shown in Table 1 in single-cell culture was examined, CP2 30 showed a level of expression $<1 \%$ compared to the wild-type construct (expression was not detectable even after a long exposure; oligonucleotides containing the pst-cath/CP2 


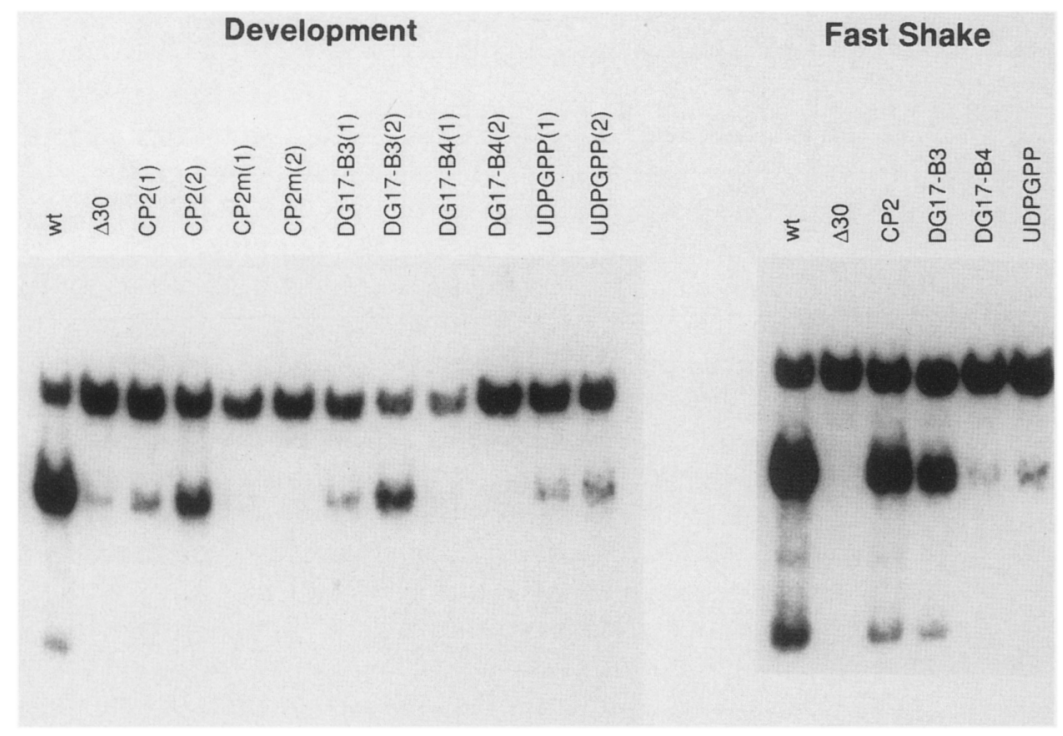

Figure 5. Rescue of pst-cath/CP2 promoter activity by insertion of G/C-rich oligonucleotides. Constructs containing G/C-rich oligonucleotides inserted into the BamHI site of the $C P 2 \Delta 30(\Delta 30)$ transformation vector were transformed into Dictyostelium Ax3 cells, and populations of transformants were selected in $10 \mu \mathrm{g} / \mathrm{ml} \mathrm{G418.} \mathrm{The} \mathrm{transformed} \mathrm{plasmids}$ contain, respectively, one (1n) and two (2n) copies of the various G/C-rich oligonucleotides, according to the nomenclature used in Fig. 1. Transformants carrying one- and twocopy oligonucleotide clones were analyzed at $15 \mathrm{hr}$ of development, and the two-copy clones were analyzed by CAMP induction in the single-cell culture (fast shake). A construct carrying $\sim 900 \mathrm{bp}$ of the wild-type upstream region of the pst-cath/CP2 is included (wt). Total cellular RNA was isolated at the tipped aggregate stage ( $15 \mathrm{hr}$ into development) or from cAMPinduced single-cell cultures, and samples containing $5 \mu \mathrm{g}$ of RNA were separated on $1.2 \%$ formaldehyde gels. RNA blots were hybridized with a probe representing the $5^{\prime}$ half of the pst-cath/CP2 gene. The larger mRNA species represents expression of the endogenous pst-cath/CP2 gene, and the smaller RNAs correspond to the minigene transcription products. The smallest of the three RNAs is thought to result from primative premature termination within the CP2 minigene-coding region (see Hjorth et al. 1989). DNA blot analysis was done on all transformed populations. The DNA copy of the transformed gene was $\sim 40$ copies per cell for all populations.

and DG17-B3 sequences greatly enhanced expression ( $>50$-fold) and the CP1 two-copy element similarly stimulated expression very well, although to a level that is reproducibly slightly less than the pst-cath/CP2 and DG17-B3 elements (see Fig. 5). Both of the DG17-B4 and UDPGPP constructs stimulated expression of the minigene to low levels. The relative level of stimulation over $C P 2 \triangle 30$ is unknown, as expression from CP2 30 is below the detectable level.

\section{Effect of point mutations in the CP1 element}

We analyzed the CP1 G-rich element in further detail by the introduction of single-point mutations that affect specific $\mathrm{G}$ residues. In vitro binding of nuclear proteins to two such mutants, $C P 1(6 \mathrm{~m})$ and $C P 1(50 \mathrm{~m})$, was tested by use of fragments that contain tandemly repeated copies of wild-type or mutant CP1 G-rich elements. The analysis showed that $C P 1(6 \mathrm{~m})$ had a very low level of binding, whereas binding to the mutant $C P 1(50 \mathrm{~m})$ was below the detectable limits of this assay (data not shown). These same elements were analyzed further for promoter function in vivo in multicellular development and following cAMP induction in shaking culture. The results shown in Figure 6 again demonstrate a direct correlation between relative affinity for binding in vitro and the ability to confer regulated expression in vivo. In these experiments, the CP2 330 parental construct showed no detectable expression during multicellular development $(C P 2 \Delta 30$ had $4 \%$ activity in the experiments shown in Fig. 5) or in fast shake plus cAMP. Two copies of $C P 1$ showed good induction under both experimental conditions. Two copies of $C P 1(6 \mathrm{~m})$ showed $0.5 \%$ and $1 \%$ activity of the wild-type vector in the multicellular developing and cAMP-induction assays, respectively. (Note that $C P 2 \triangle 30$ was $<1 \%$ for both of these assays in these experiments.) One copy gave $\sim 1 \%$ activity in the cAMP-induction assay (not done for multicellular development assay|. One and two copies of $C P 1(50 \mathrm{~m})$ gave no detectable activation in either assay.

Additional CP1 mutant elements were analyzed in vivo in both single-cell culture conditions and during multicellular development. The above results and other data (A. Hjorth and R.A. Firtel, unpubl.) indicated that many of the mutants we wished to examine yielded a stimulation in vivo below levels that could be accurately quantitated. Because our results with wild-type CP1 element suggested that a dimer is required for efficient expression, we decided to use constructs containing a tandem arrangement with a mutant copy of the $C P 1$ oligonucleotide located immediately upstream of a wild-type copy. The data for some of these experiments are shown in Figure 6; the results are summarized in Table 1 . As can be seen, $C P 1(50 \mathrm{~m})$, in association with a copy of the wild-type $C P 1$ sequence, shows a stimulation over two copies of $C P 1(50 \mathrm{~m})$. The low level of expression in relationship to that of two constructs carrying wild-type $C P 1$ copies suggests that, at the very least, three to four specific $G$ residues (as indicated, GGAATGGGGGTT) are essential for activity of the element.

We also examined the ability of wild-type and mutant CP1 elements to compete for binding to the pst-cath/ 
CP2, DG17-B3, and CP1 elements in vitro (Fig. 7). In these experiments, a 15 -fold excess of a ligated competitor was used in contrast to the 50-fold competitor used in Figure 2. As shown previously (Figs. 2 and 3), a CP1

Table 1. Quantitation of oligonucleotide-directed pst-cath/CP2 minigene expression

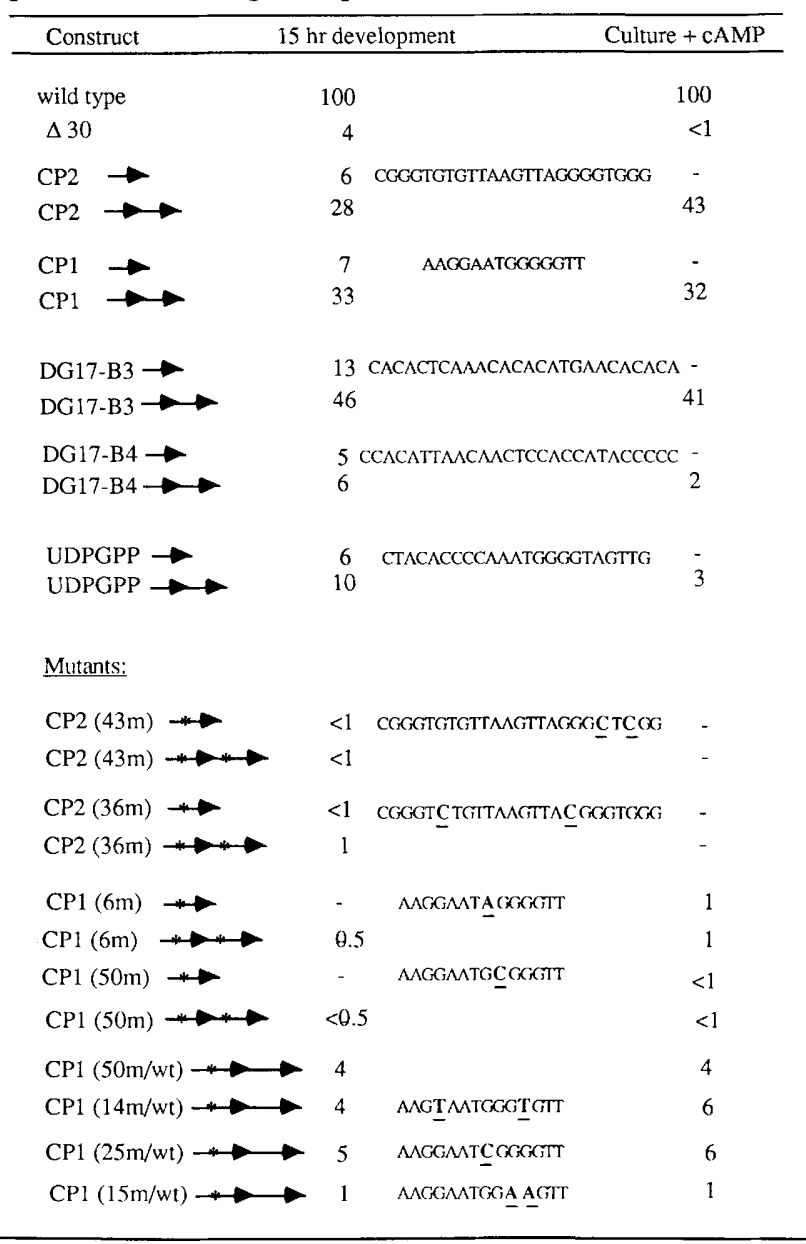

The orientation and the number of oligonucleotides present in the individual constructs are indicated by arrows. The asterisks $1 * \mid$ represent the positions of mutations within the oligonucleotides. The nature of mutant residues within the pst-cath/CP2 and $C P 1$ oligonucleotides is indicated by underlining and boldface letters. Autoradiographs of RNA blots were scanned by laser densitometry, and the level of expression of the transformed mRNA species was normalized relative to the level of endogenous pst-cath/CP2 mRNA in the same sample. Expression is calculated as a percentage of that seen for the construct carrying $\sim 900$ bp of upstream sequences (wt). In the experiments shown in Fig. 5 for multicellular development, the $C P 2 \Delta 30$ had a level of expression that was $4 \%$ of the control vector. In the experiments shown in Fig. 6 , there was no detectable $C P 2 \Delta 30$ expression. In other experiments, $C P 2 \Delta 30$ expression ranged from undetectable to a maximum of $4 \%$ from experiment to experiment and transformation to transformation. The other results showed only small quantitative changes between experiments and no qualitative differences. Wild-type recorder gene and $C P 2 \Delta 30$ were included in each assay. In all cases, cells developed normally and had normal morphology at the time the cells were harvested. dimer competes for binding of nuclear factor(s) to the $C P 2$ dimer oligonucleotide. The mutant $C P 1(6 \mathrm{~m})$ shows a low level of competition (approximately one-third that of wild-type $C P 1)$, whereas $C P 1(50 \mathrm{~m})$ shows no detectable competition for $C P 1$ probe binding. When the relative levels of competition for $C P 2$ and $D G 17$-B3 probes were examined, we see that the wild-type dimer $C P 1$ oligonucleotide competed as expected from our previous estimate of relative binding affinities of the three sequences (Fig. 3). Also, the two CP1 mutant oligonucleotides show a decreased ability to compete for the binding of the nuclear factor(s) to the CP2 and the DG17-B3 elements. Two copies of $C P 1(50 \mathrm{~m})$ show no competition, whereas $C P 1(6 \mathrm{~m})$ again shows a low level of competition. UDPGPP and DG17-B4 were also examined and showed similar results (data not shown).

Previously, we have shown that GBF-binding activity can be induced in the single-cell cAMP induction assay and that this induction is inhibited by prior or concomitant addition of cycloheximide (Hjorth et al. 1989). This activity is decreased as a result of competition by a $30-$ fold molar excess of ligated CP2 wild-type 43-mer (43w) but not by the ligated mutant CP2 $43-\mathrm{mer}(43 \mathrm{~m})$. We have now tested the $C P 1$ oligonucleotides and have shown that wild-type $C P 1$ oligonucleotide competes, whereas $C P 1(50 \mathrm{~m})$ shows no competition and $C P 1(6 \mathrm{~m})$ shows a very slight competition (data not shown). These in vivo and in vitro data suggest that the $C P 1 \mathrm{G}$-rich element is functionally homologous to the pst-cath/CP2 GBRE, as had been proposed previously (Pears and Williams 1988) and that the same factor or family of related factors interacts with the pst-cath/CP2 and $C P 1$ elements in vitro, thus playing a regulatory role in the expression of both of these genes in vivo.

\section{Structural aspects of DNA-protein interactions}

Although previous data suggest that the pst-cath/CP2, $C P 1$, and DG17-B3 G/C-rich elements represent functionally similar promoter elements and that these sequences are recognized by the same or a related set of factors in vitro, there is no substantial structural similarity between these elements besides a strikingly high $\mathrm{G} / \mathrm{C}$ content relative to the surrounding DNA sequences. To analyze DNA-protein interactions in more detail, we performed DMS methylation interference assays to pinpoint essential G residues for the pst-cath/ $C P 2$ and the DG17-B3 elements. Methylation interference data for the G-rich strand of the DG17-B3 element (Fig. 8A; the opposite strand from that shown in Fig. 1) were analyzed by densitometry, and the data were normalized to a $G$ residue located within the polylinker sequence that surrounds the DG17-B3 oligonucleotide. The gel showing the methylation interference analysis of the pst-cath/CP2 G-box has been published previously (Hjorth et al. 1989); a densitometer tracing of that gel is presented here as a comparison to the DG17B3 interference pattern (see Fig. 8B). The results from this analysis do not reveal any striking similarities in the protection pattern for the two sequences, which is not unexpected because the sequences are so different. 


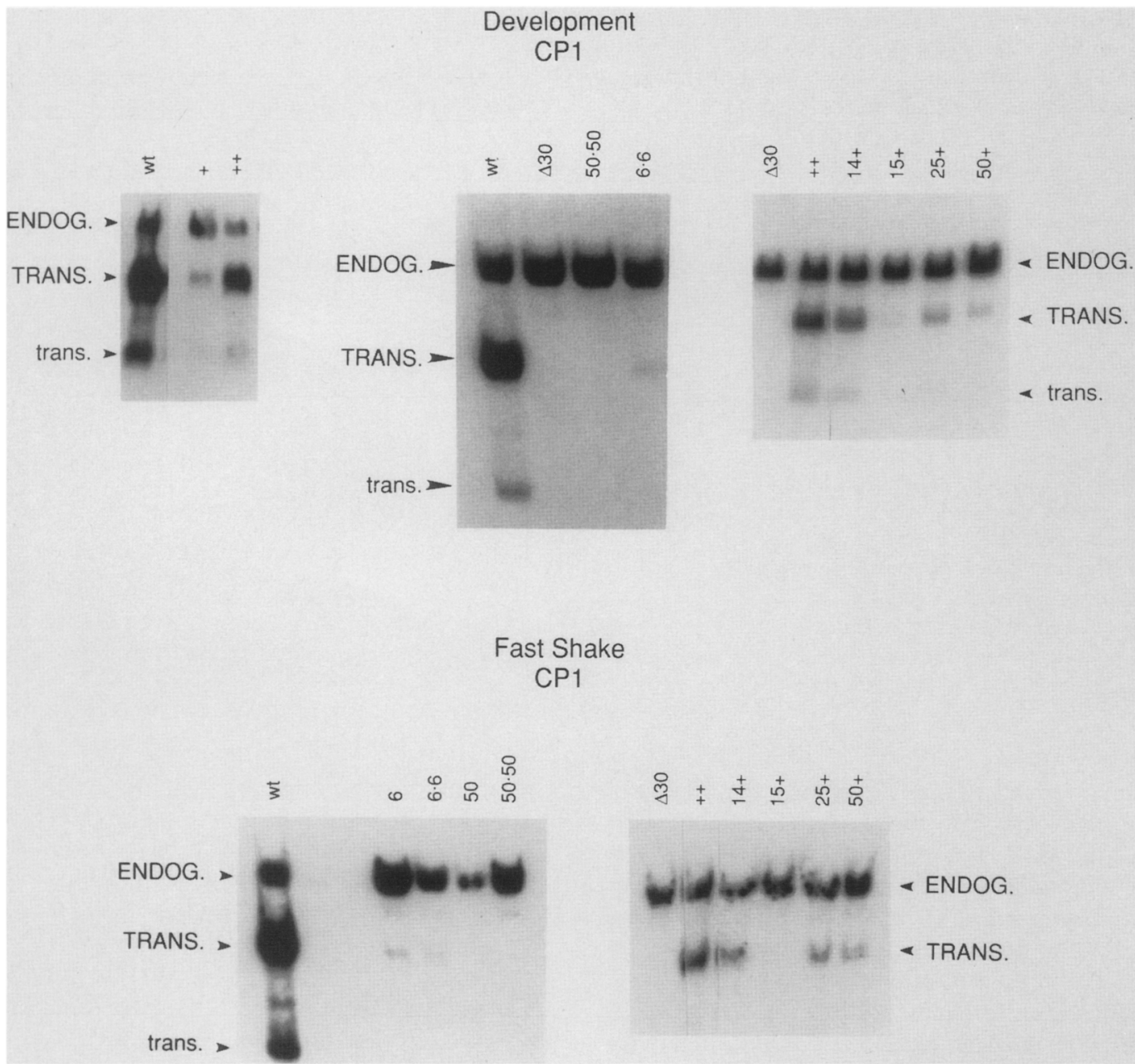

Figure 6. $C P 1$ oligonucleotide-directed expression of the pst-cath/CP2 minigene. The constructs analyzed here contained one or two copies of wild-type $(+\mid$ and mutant $(6,14,15,25,50) C P 1$ oligonucleotides. The sequence of these CP1 oligonucleotides is presented in Fig. 1 and Table 1. The tandemly arranged oligonucleotides were either identical in sequence (e.g., ++ and 6.6$)$ or consisted of a wild-type oligonucleotide inserted immediately downstream from a mutant copy (e.g., $14+, 50+$ ). Dictyostelium transformants were analyzed for $\sim 15 \mathrm{hr}$ during development (development) or induced with cAMP in shaking culture (fast shake). Northern blots were prepared from $1.2 \%$ formaldehyde-agarose gels and hybridized to a probe containing a 5 ' portion of the pst-cath/CP2 gene. The arrowheads indicate the signal corresponding to the endogenous pst-cath/CP2 gene and the smaller species representing the transformed minigene mRNA. An additional small species of mRNA (trans.) is thought to result from premature termination within the CP2 minigene-coding region (Hjorth et al. 1989). Each autoradiograph represents samples from the same experiment; unrelated lanes were removed in some cases.

Because protection of a given $G$ residue in this assay is indicative of that particular $G$ residue being essential for complex formation, binding of the nuclear factor(s) to both of these oligonucleotides appears to involve a region $\sim 20 \mathrm{bp}$ long. For both of the binding sites, the most strongly protected $G$ residues are spaced $\sim 10$ bp apart and the appearance of nonessential $G$ residues within this $\sim 20$-bp region may reflect predominant binding of the protein to only one side of the DNA helix. Attempts were made to examine the DMS methylation interference pattern of a tandem CP1 dimer. However, the results indicated that very little, if any, probe contained methylating $G$ residues was in the bound fraction, suggesting that methylation of any of the $G$ residues may substantially reduce the binding affinity to the protein forming the complex in vitro.

\section{Discussion}

In this paper we present a functional analysis of known and putative promoter elements for a group of four cAMP-inducible, aggregate-stage genes in Dictyostelium. We have shown that $\mathrm{G} / \mathrm{C}$-rich oligonucleotides from the promoter regions of these genes reconstitute expression when inserted into a pst-cath/CP2 GBRE deletion construct $(C P 2 \Delta 30)$, either in multicellular aggregates or in shaking culture in response to cAMP. Our results extend previous analysis on the pst-cath/CP2 and 
Figure 7. Competition analysis using $C P 1$ wild-type and mutant elements. Labeled DNA fragments containing two cloned copies of $C P 2, C P 1$, and DG17-B3 oligonucleotides, respectively (see Fig. 1), were incubated with nuclear extract and analyzed on native acrylamide gels. (NE) A control containing no nuclear extract. Specific competitors were present in the binding assay in 15-fold molar excess (in addition to a 500 -fold excess of poly[d(I-C)] as follows: $(-)$ No specific competitor; $(++)$ a fragment containing two copies of the wildtype CP1 G-rich element; $(6.6$ and 50.50) twocopy fragments containing the $C P 1(6 \mathrm{~m})$ and the $C P 1(50 \mathrm{~m})$ mutant oligonucleotides, respectively (see Table 1). The result of the analysis that includes the $C P 1$ element as a binding substrate is presented twice. (A) A short exposure of the gels where all three probes are exposed together; $(B)$ a longer exposure of the samples containing the CP1 sequence as a probe. This is induced because the specific activity, and thus, total input counts of the CP1 probe, are less than those of the other two probes.

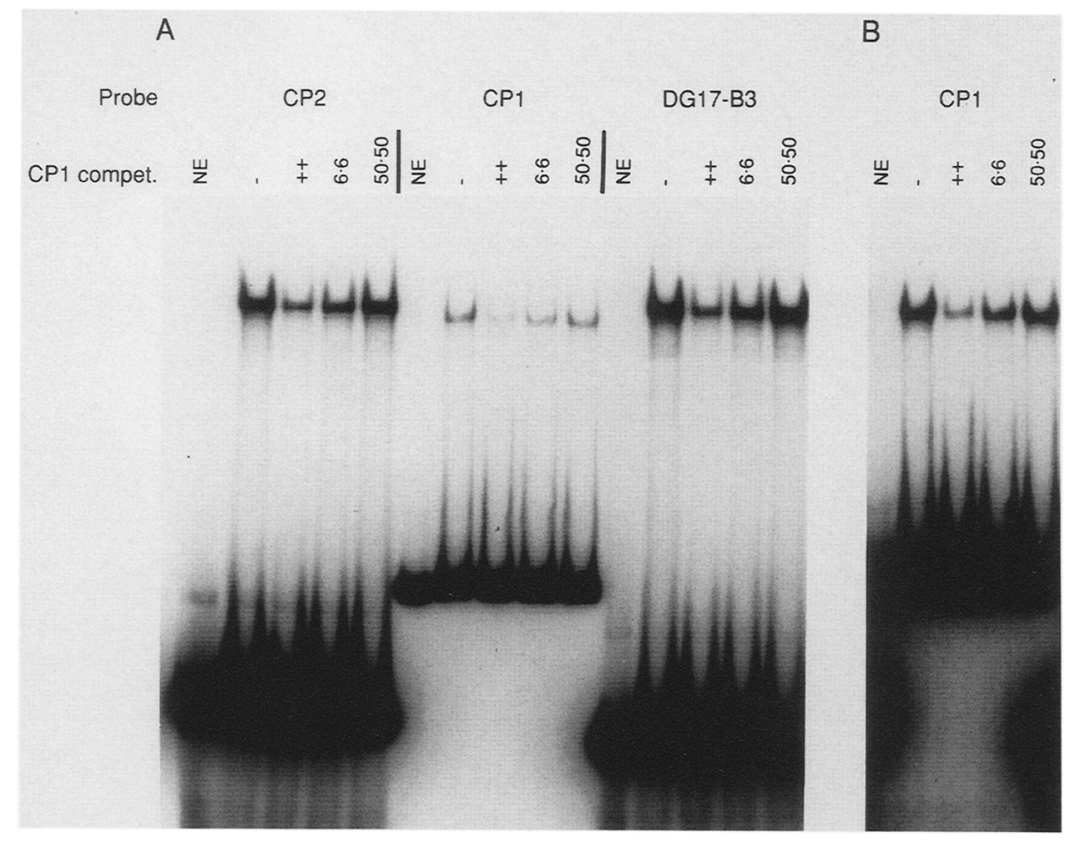

$C P 1$ G-rich elements and show that one of the DG17 G/C-rich elements can also function in this way. Surprisingly, another DG17 G-rich element, which is remarkably homologous to an inverted $C P 1 \mathrm{G}$-rich element, and a palindromic G/C-rich UDPGPP element required for cAMP-inducible expression both yielded a relatively small increase in reporter gene expression. It is possible that the differences in activity seen between these two elements and DG17-B4 and UDPGPP may reflect changes in essential $G$ residues or that these latter two elements are not functionally related to the $C P 1$ and pst-cath/CP2 G-rich elements. In the case of DG17-B3 and pst-cath/CP2, substantially higher levels were seen with two copies of the element relative to that shown with a single element, similar to observations with a number of elements from other systems (e.g., Stuart et al. 1984; Bienz and Pelham 1986; Comb et al. 1986).

In vivo results (see Fig. 6; Table 1; Pears and Williams 1988) and particularly our in vitro binding data suggest that the CP1 G-rich sequence behaves as a half-element in certain assays. A single copy shows negligible binding to proteins in our nuclear extract, whereas a dimer shows strong binding. The sequence structure of the pstcath/CP2 GBRE suggests that the element is bipartite with two nucleotide mismatches between the two halves. Neither half of the element is capable, itself, of inducing expression in vivo (Datta and Firtel 1987; Hjorth et al. 1989) nor forming a complex in vitro using a gel shift assay (A. Hjorth and R.A. Firtel, unpubl.), and complex formation with a pst-cath/CP2 GBRE results in a single band in a gel mobility-shift assay. Moreover, methylation interference studies have indicated that $G$ residues located within both domains are required for DNA binding. Taken together, these results suggest that the $C P 1$ and pst-cath/CP2 elements represent single functional sequence elements that interact with GBF or a family of related factors. This bipartite structure may suggest that the nuclear factor(s) interacts with these elements as either a homo- or a heterodimer. In addition, the UDPGPP element is palindromic in structure, whereas the $D$ G17-B3 element appears to have a repeated structure.

Previously, we have shown that oligonucleotides containing point mutations in the pst-cath/CP2 GBRE that significantly reduced binding to GBF in vitro also cannot complement promoter activity in vivo (Hjorth et al. 1989|. This represented strong molecular genetic evidence that the protein detected in gel shifts in vitro functions in vivo to activate gene expression from this element. In this report we show that two oligonucleotide point mutations affecting $\mathrm{G}$ residues in the $C P 1$ element, $C P 1(6 \mathrm{~m})$ and $C P 1(50 \mathrm{~m})$, complement expression in vivo either very poorly $[C P 1(6 \mathrm{~m})]$ or not at all $[C P 1(50 \mathrm{~m})]$ and that their affinity of interaction with putative trans-acting factors in vitro parallels their activity in vivo. Both of the mutated $C P 1$ oligonucleotides restored some activity in vivo when present in association with a wild-type element. This level of induction is substantially higher than that seen with two copies of the mutant elements. Other $C P 1$ oligonucleotides containing one or two point mutations were similarly examined in combination with a wild-type element, allowing us to determine the importance of specific nucleotides for in vivo function. These results similarly support the premise that two copies of the CP1 element are required for induction and indicate that certain point mutations can reduce activation substantially.

DNA-binding and competition studies, using partially purified nuclear extracts, suggest that a common nuclear factor, or family of related factors, interacts with all of the $\mathrm{G} / \mathrm{C}$-rich sequence elements we have analyzed. Moreover, we have observed a direct correlation be- 

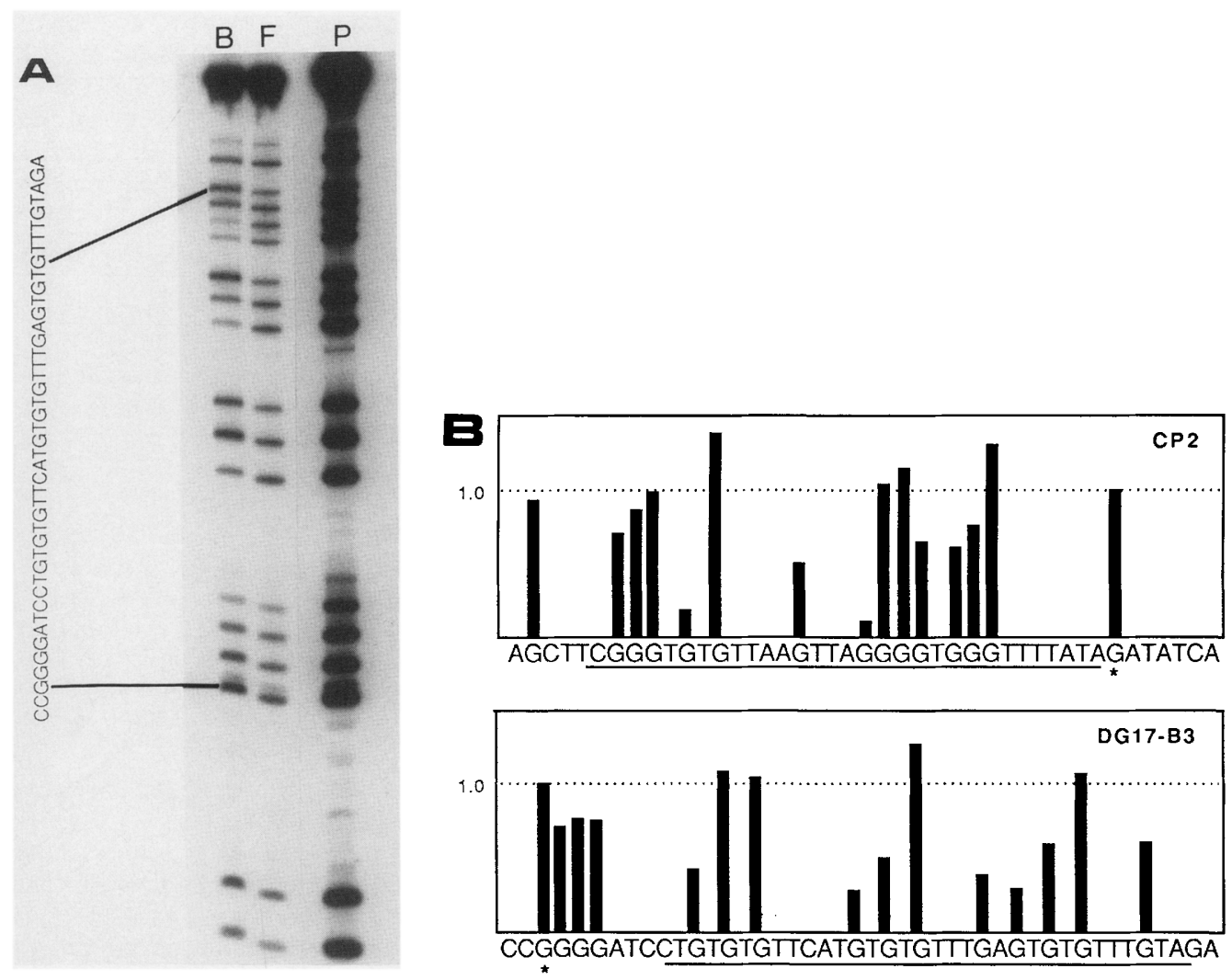

Figure 8. Quantitation of a DMS interference analysis using high-affinity binding sites. (A) DMS interference assay with DG17-B3. End-labeled DNA was pretreated at low levels with DMS, and the ability of the DNA to form protein complexes was examined. The DNA-protein complexes and the free DNA were extracted from a mobility-shift gel, cleaved at the methylated G residues by piperidine, and analyzed on denaturing gels (see Materials and methods). If methylation of a particular $\mathrm{G}$ residue prevents binding, then that particular molecule will be underrepresented in the protein-complexed form of the DNA (bound) relative to the free form of DNA. Thus, by comparing the G-specific cleavage pattern in the bound and unbound forms of DNA, a methylation interference pattern is obtained and $G$ residues required for binding are identified. Nuclear extract was incubated with uniquely end-labeled and partially methylated DNA fragments containing a single copy of the pst-cath/CP2 43-mer oligonucleotide or the DG17-B3 37-mer oligonucleotide, respectively (Fig. 1). Bound and free DNAs were isolated, following separation on native acrylamide gels, cleaved preferentially at methylated $\mathrm{G}$ residues, and analyzed on a $7 \mathrm{M}$ urea/ $10 \%$ acrylamide gel. The autoradiograph represents the experiment using the DG17-B3 sequence as a binding substrate. A gel representing an experiment using the $p s t-c a t h / C P 2$ sequence has been published previously (Hjorth et al. 1989). Note that the DG17-B3 probe contains four additional $G$ residues at the 5 ' end derived from the polylinker. The $\mathrm{G}$ residues have no effect on binding because probes lacking these residues have the same relative affinity for the nuclear factor(s), the same relative behavior in competition studies, and the same pattern in gel mobility shifts (data not shown). (B) Quantitation of DMS interference assays with pst-cath/CP2 and DG17. Quantitation of the protection pattern was performed by laser densitometry. The difference in total counts of the free versus complexed form of DNA was normalized relative to the intensity of a $G$ residue $(*)$ located within the polylinker sequence. Protection/enhancement of individual $G$ residues was then calculated for each band as the intensity of that particular band in the complexed form of DNA, relative to the intensity of the corresponding band in free DNA. The relative intensity for each $\mathrm{G}$ residue is indicated by the height of the bars in this diagram. The underlined regions represent Dictyostelium sequences.

tween the affinity of the nuclear factor for the various G/C-rich wild-type and mutant oligonucleotides and the ability of these elements to complement gene expression in vivo. On the basis of these data, we propose that formation of the CP1, pst-cath/CP2, and DG17-B3 DNAprotein complexes involves a common protein, GBF, or a family of related proteins with similar binding affinities, and that $C P 1$, pst-cath/CP2, and DG17, all of which are cAMP-inducible, are regulated by a common molecular mechanism that involves developmentally regulated factors such as GBF.

Because of the relatively low level of promoter ac- tivity that is conferred by insertion of the UDPGPP G/C-rich element, we are not as confident that the same factor, or set of factors, also mediates expression of this gene; yet, we do see a consistent pattern for the UDPGPP G/C-rich element with respect to binding in vitro and gene activation in vivo. Although the UDPGPP element appears to form a single complex with our partially purified nuclear extract, we cannot exclude the possibility that factors in addition to GBF are associated with the complex and that the association of such factors depends on the proper promoter context for activity in vivo. Moreover, the relative level of cAMP 
inducibility of UDPGPP expression is consistent with the putative low binding affinity to GBF in vitro. Whether this element depends on GBF in vivo in the context of the UDPGPP promoter is not known.

The function of the DG17-B4 element is also unclear because it functions rather poorly both in vitro and in vivo. Possibly, it may confer weak stimulation, perhaps by acting in combination with the DG17-B3 element.

Methylation interference studies on the two strongest G/C-rich elements identify multiple sites of interaction, extending over a region of $\sim 20 \mathrm{bp}$ for both binding sites and suggest that there is substantial flexibility in DNAbinding domain(s) of the trans-factor(s) that allows it to recognize diverse binding sites. There are other known examples of yeast and mammalian trans-acting factors that appear to interact with very dissimilar sequence elements, some with comparable and some with reduced affinity (e.g., Pfeifer et al. 1987; Landschulz et al. 1988; Garcia-Blanco et al. 1989; Mercurio and Karin 1989). It seems possible that GBF also is able to interact with a diverse range of $\mathrm{G} / \mathrm{C}$-rich sequence elements and still maintain sufficient sequence specificity; alternatively, GBF may be a common factor, and the interaction with $\mathrm{G} / \mathrm{C}$-rich sequences in vitro may depend on the association of more than one protein, one of which is GBF. Other studies have also pointed out that the binding affinity of GBF, or related nuclear factors for these various sequences, is not exceptionally high and that the protein has a very fast off-rate (A. Hiorth, N.C. Khanna, and R.A. Firtel, unpubl.), which is consistent with the observations described in Figure 4. High-affinity DNA-protein interactions may not be possible with the diverse nature of the sequence elements with which this protein appears to interact. In vivo, the affinity of DNA-protein complex formation may be considerably enhanced by the participation of additional proteins. In the case of pst-cath/CP2, we know that the promoter element required for developmental and cAMP induction extends over $80 \mathrm{bp}$; conceivably additional trans-acting factors associate with various sequences within this region and thereby serve to stabilize the binding of GBF to the region encompassing the G-box. In support of this, we have shown previously that a region of $\sim 180 \mathrm{bp}$ from the pst-cath/CP2 gene containing this 80 -bp regulatory region and only the single pst-cath/CP2 G-box shows three specific bands on a gel shift assay, all of which are competed with a GBRE-containing oligonucleotide (Hjorth et al. 1988). These results suggest that additional factors may be involved with some of these complexes, but the formation of any of the complexes requires GBF or related factors. We believe that the other genes examined here may have regulatory sequence elements that serve a similar function. Further purification of GBF followed by the cloning and expression of the GBF-encoding gene should help to clarify these issues.

\section{Materials and methods}

Growth and development of Dictyostelium cells

Dictyostelium KAx-3 cells were grown in HL-5 medium and harvested at a density of $10^{6}$ to $3 \times 10^{6}$ cells $/ \mathrm{ml}$. The cells were washed in phosphate buffer and plated for multicellular development on buffer-saturated filters. To assay cAMP induction, cells were resuspended at $5 \times 10^{6}$ cells $/ \mathrm{ml}$ and shaken for $5 \mathrm{hr}$, after which one-half of the cells were given $300 \mu \mathrm{M}$ cAMP plus $100 \mu \mathrm{M}$ CAMP every $2 \mathrm{hr}$, and the other aliquot was shaken in the absence of cAMP, as described previously (Mehdy et al. 1983; Datta and Firtel 1988; Hjorth et al. 1989|.

\section{Nuclear extract}

The nuclear extract was prepared from cells at $15 \mathrm{hr}$ of development, as described previously (Hjorth et al. 1989). The crude nuclear extract was concentrated by a $60 \%$ ammonium sulfate precipitation in extraction buffer [ $25 \mathrm{mM}$ HEPES ( $\mathrm{pH} 7.5$ ), 12.5 $\mathrm{mM} \mathrm{MgCl}, 100 \mathrm{~mm} \mathrm{KCl}, 0.1 \mathrm{~mm}$ EDTA, $1 \mathrm{~mm} \mathrm{DTT}, 20 \%$ (vol/ vol) glycerol, and $0.1 \%$ Triton $X-100$ ] and subsequently heat treated at $80^{\circ} \mathrm{C}$ for $2 \mathrm{~min}$ in the presence of $0.1 \mathrm{mg} / \mathrm{ml} \mathrm{BSA}$ (Sigma), which results in a purification of $\sim 20$-fold. The extract was frozen in aliquots at $-85^{\circ} \mathrm{C}$; the same extract preparation was used for all binding studies.

\section{Isolation and analysis of Dictyostelium transformants}

Dictyostelium cells were transformed by electroporation, as described previously (Howard et al. 1988; Dynes and Firtel 1989). Transformants were selected on plates in the presence of 10 $\mu \mathrm{g} / \mathrm{ml} \mathrm{G418}$ and later transferred to shaking culture. The copy number of transformed plasmids was determined by DNA blot analysis of genomic DNA, following digestion with HindIII and Asp 718, which releases a fragment containing the 5 ' portion of the pst-cath/CP2 minigene from the remainder of the transformation vector. DNA blots were hybridized with probes made from fragments also used for RNA blot analysis. Purified plasmid DNA of the transformation vector $C P 2 \Delta 30$, also digested with HindIII and Asp718, was used as standard for estimation of copy number (Nellen et al. 1986).

\section{RNA analysis}

Transformants were plated for multicellular development and harvested when the cells reached the early finger stage $(\sim 15 \mathrm{hr})$. For single-cell assay, cells were washed and placed in shaking culture in MES-PDF (buffered salts) for $6 \mathrm{hr}$, followed by an additional $6 \mathrm{hr}$ with or without the addition of $300 \mu \mathrm{M}$ cAMP (Mehdy et al. 1983). This has been shown to induce aggregatestage gene expression (see Mehdy et al. 1983). RNA was isolated, separated on $1.2 \%$ agarose-formaldehyde gels, and transferred to nylon membranes (Mann and Firtel 1987). The RNA blots were hybridized using a ${ }^{32}$ P-labeled random oligonucleotide-primed probe prepared from a fragment containing $5^{\prime}$ and coding sequence $(-742$ to +386$)$ of the pst-cath/CP2 minigene (Pears and Williams 1988; Hjorth et al. 1989). The hybridization probe is complementary to both the endogenous and the transformed minigene. Quantitation of expression from the transformed pst-cath/CP2 vectors was performed by densitometry of autoradiogrphs and normalized to the level of expression of the endogenous gene in each sample. Multiple exposures were quantitated to assure that the exposure levels were linear.

\section{Transformation vector}

The vector used for these studies is a pst-cath/CP2 minigene construct that contains a 750 -bp deletion in the coding region, the transcription of which results in a minigene mRNA that is distinguishable in size from the endogenous mRNA. A con- 
struct containing $\sim 900$ bp of upstream sequence was used as a control for full levels of expression of the minigene; this construct is designated wt (wild-type) in the transformation studies. The $C P 2 \Delta 30$ vector (designated $\Delta 30$ in figures) contains a 30-bp deletion that removes both of the G-boxes from the pstcath/CP2 regulatory region and has a $B a m H I$ insertion site at this position. These vectors have been described previously (Pears and Williams 1987, 1988; Hjorth et al. 1989).

\section{Purification and cloning of oligonucleotides}

Oligonucleotides were purified by thin-layer chromatography on silica plates (Alvarado-Urbina et al. 1981). Complementary oligonucleotides were annealed by gradual cooling $\left(80-22^{\circ} \mathrm{C}\right)$ in kinase buffer (Maniatis et al. 1982), phosphorylated with polynucleotide kinase, and ethanol-precipitated in the presence of $2.5 \mathrm{M} \mathrm{NH}_{4}$ acetate. Following an additional ethanol precipitation, the oligonucleotides were reannealed in ligation buffer (Maniatis et al. 1982) and ligated at $15^{\circ} \mathrm{C}$ for $34 \mathrm{hr}$. Self-ligated oligonucleotides were rephosphorylated with polynucleotide kinase and ligated to both the linearized, dephosphorylated pUC1 8 and the $C P 2 \Delta 30$ transformation vectors. All constructs were tested for the presence, identity, and orientation of inserted oligonucleotides by DNA sequencing. Most of the oligonucleotides were synthesized with a $\mathrm{BamHI}$ restriction site on one end $\left(5^{\prime}\right.$ orientation as in the gene) and a BgIII site at the other end, so that cloning into the BamHI site of the vector recreated the BamHI site at one end only, facilitating analysis of the orientation of the oligonucleotide prior to sequencing. The pst-cath/CP2 43-mer (Fig. 1) and the CP1 30-mer used to create the DNA-binding fragment (same genomic sequence as the CP1 28-mer; Fig. 1) contained HindIII sites at the ends.

\section{Gel mobility-shift assays}

Oligonucleotide-containing DNA fragments were prepared by digestion of the clones with Asp718 and PstI (CP2, DG17-B3, B4, and UDPGPP) or PvuII and PstI (CP1). Fragments were separated on $6 \%$ acrylamide gels, eluted by diffusion, and dephosphorylated with alkaline phosphatase. The concentration of the fragments was estimated by ethidium bromide staining and compared to a known standard [HaeIII digest of pSP72 (Promega)]. When used as probes, DNA fragments were end-labeled with $\left[\gamma^{-32} \mathrm{P}\right] \mathrm{ATP}$ and polynucleotide kinase and purified on Sephadex G-50 spin columns. Gel mobility-shift assays were performed as described previously in a final volume of $205 \mu \mathrm{l}$, using, in the standard assay, $105 \mu$ of heat-treated extract, 0.2 $\mathrm{ng}$ of labeled probe, and a 500 -fold excess of nonspecific competitor poly[d(I-C)] (Hjorth et al. 1989). Specific competitors were added in molar excess relative to the probe, as indicated in the figures. Quantitation of complex formation was performed using LKB-Ultroscan densitometry of autoradiographs. Multiple exposures were quantitated to assure linearity of the exposures.

\section{DMS interference assays}

The probes used in this assay contain a single copy of the pstcath/CP2 43-mer or the DG17-B3 37-mer oligonucleotide. The probes were prepared by labeling at one end with $\left[\gamma^{-32} \mathrm{P}\right] \mathrm{ATP}$ and polynucleotide kinase. Following purification on Sephadex G-50 columns, the probes were methylated with DMS as described previously (Hjorth et al. 1989). The partially methylated probes were incubated with nuclear extract using standard binding conditions and electrophoretically separated on native acrylamide gels. Free and bound forms of DNA were visualized by exposure of wet gels. DNA was excised, purified by elution onto NA45 paper (Schleicher \& Schuell), concentrated by ethanol precipitation, and cleaved with $1 \mathrm{M}$ piperidine at $90^{\circ} \mathrm{C}$ for $30 \mathrm{~min}$. The samples were washed and lyophilized more than three times and resuspended in $90 \%$ formamide and $1 \mathrm{mM}$ EDTA, and approximately equal counts of free and bound DNA were analyzed by electrophoresis on $7 \mathrm{M}$ urea/ $10 \%$ acrylamide gels.

\section{Acknowledgments}

We thank Dr. Navin Khanna for his help in making the nuclear extract, Gavin Schnitzler for his stimulating interest in this project, and M. Geoffrey Rosenfeld, M. Karin, and R. Hori for critical suggestions on the manuscript. This work was supported by a U.S. Public Health Service grant to R.A.F. and by the Imperial Cancer Research Fund.

\section{References}

Alvarado-Urbina, G., G.M. Sathe, W. Liu, M.F. Gillen, P.D. Duck, R. Bender, and K.K. Ogilvie. 1981. Automated synthesis of gene fragments. Science 214: 270-274.

Barklis, E. and H.F. Lodish. 1983. Regulation of Dictyostelium discoideum mRNAs specific for prespore and prestalk genes. Cell 32: 1139-1148.

Bienz, M. and H.R.B. Pelham. 1986. Heat shock regulatory elements function as an inducible enhancer in the Xenopus hsp70 gene and when linked to a heterologous promoter. Cell 45: 753-760.

Comb, M., N.C. Birnberg, A. Seasholtz, E. Herbert, and H.M. Goodman. 1986. A cyclic AMP- and phorbol ester-inducible DNA element. Nature 323: 353-356.

Datta, S. and R.A. Firtel. 1987. Identification of the sequences controlling cyclic AMP regulation and cell-type-specific expression of a prestalk-specific gene in Dictyostelium discoideum. Mol. Cell. Biol. 7: 149-159.

1988. An $80-\mathrm{bp}$ cis-acting regulatory region controls cAMP and development regulation of a prestalk gene in Dictyostelium. Genes Dev. 2: 294-304.

Datta, S., R.H. Gomer, and R.A. Firtel. 1986. Spatial and temporal regulation of a foreign gene by a prestalk-specific promoter in transformed Dictyostelium discoideum. Mol. Cell. Biol. 6: $811-820$.

Driscoll, D.M. and J.G. Williams. 1987. Two divergently transcribed genes of Dictyostelium discoideum are cyclic AMPinducible and coregulated during development. Mol. Cell. Biol. 7: 4482-4489.

Dynes, J. and R.A. Firtel. 1989. Molecular complementation of a genetic marker in Dictyostelium using a genomic library. Proc. Natl. Acad. Sci. (in press).

Firtel, R.A. and A.L. Chapman. 1990 A role for cAMP-dependent protein kinase in early Dictyostelium development. Genes Dev. (in press).

Firtel, R.A., P.J.M. van Haastert, A.R. Kimmel, and P.N. Devreotes. 1989. G protein linked signal transduction pathways in development: Dictyostelium as an experimental system. Cell 58: 235-239.

Garcia-Blanco, M.A., R.G. Clerc, and P.A. Sharp. 1989. The DNA-binding homeo domain of the Oct-2 protein. Genes Dev. 3: 739-745.

Gomer, R.H., S. Datta, and R.A. Firtel. 1986a. Cellular and subcellular distribution of a cAMP-regulated prestalk protein and prespore protein in Dictyostelium discoideum: A study on the ontogeny of prestalk and prespore cells. J. Cell. Biol. 103: 1999-2015. 
Gomer, R.H., D. Armstrong, B.H. Leichtling, and R.A. Firtel. 1986b. cAMP induction of prespore and prestalk gene expression in Dictyostelium is mediated by the cell surface cAMP receptor. Proc. Natl. Acad. Sci. 83: 8624-8628.

Haribabu, B. and R.P. Dottin. 1986. Pharmacological characterization of cyclic AMP receptors mediating gene regulation in Dictyostelium discoideum. Mol. Cell. Biol. 6:24022408.

Haribabu, B., A. Rajkovic, and R.P. Dottin. 1986. Cell-cell contact and cAMP regulate the expression of a UDP glucose pyrophosphorylase gene of Dictyostelium discoideum. Dev. Biol. 113: 436-442.

Haribabu, B., J. Pavlovic, and R.P. Dottin. 1988. Regulation of the rates of transcription of several specific genes in response to exogenous cAMP in Dictyostelium discoideum. Mol. Genet. 8: 1-5.

Hjorth, A., S. Datta, N.C. Khanna, and R.A. Firtel. 1988. Analysis of cis and trans elements involved in cAMP inducible gene expression in Dictyostelium discoideum. Dev. Genet. 9: 435-454.

Hjorth, A., N.C. Khanna, and R.A. Firtel. 1989. A trans-acting factor required for cAMP-induced gene expression in Dictyostelium is regulated developmentally and induced by cAMP. Genes Dev. 3: 747-759.

Howard, P.K., K.G. Ahern, and R.A. Firtel. 1988. Establishment of a transient expression system for Dictyostelium discoideum. Nucleic Acids Res. 16: 2613-2623.

Janssens, P.M.W. and P.J.M. van Haastert. 1987. Molecular basis of transmembrane signal transduction in Dictyostelium discoideum. Microbiol. Rev. 51: 396-418.

Jermyn, K.A., M. Berks, R.R. Kay, and J.G. Williams. 1987. Two distinct classes of prestalk-enriched mRNA sequences in Dictyostelium discoideum. Development 100: 745-755.

Kimmel, A. 1987. Different molecular mechanisms for cAMP regulation of gene expression during Dictyostelium development. Dev. Biol. 122: 163-171.

Kimmel, A.R. and R.A. Firtel. 1983. Sequence organization in Dictyostelium: Unique structure at the 5 '-ends of protein coding genes. Nucleic Acids Res. 11: 541-552.

Landschulz, W.H., P.F. Johnson, E.Y. Adashi, B.J. Graves, and S.L. McKnight. 1988. Isolation of a recombinant copy of the gene encoding C/EBP. Genes Dev. 2: 786-800.

Loomis, W.F., (ed.) 1975. Dictyostelium discoideum, a developmental system. Academic Press, New York.

- 1982. The development of Dictyostelium discoideum. Academic Press, New York.

Maniatis, T., E.F. Fritsch, and J. Sambrook. 1982. Molecular cloning: A laboratory manual. Cold Spring Harbor Laboratory, Cold Spring Harbor, New York.

Mann, S.K.O. and R.A. Firtel. 1987. Cyclic AMP regulation of early gene expression in Dictyostelium discoideum: Mediation via the cell surface cyclic AMP receptor. Mol. Cell. Biol. 7: 458-469.

- 1989. Two-phase regulatory pathway controls cAMP receptor-mediated expression of early genes in Dictyostelium. Proc. Natl. Acad. Sci. 86: 1924-1928.

Mehdy, M.C. and R.A. Firtel. 1985. A secreted factor and cAMP jointly regulate cell-type specific gene expression in Dictyostelium discoideum. Mol. Cell. Biol. 5: 705-713.

Mehdy, M.C., D. Ratner, and R.A. Firtel. 1983. Induction and modulation of cell-type-specific gene expression in Dictyostelium discoideum. Cell 32: 763-771.

Mercurio, F. and M. Karin. 1989. Transcription factors AP-3 and AP-2 interact with the SV40 enhancer in a mutually exclusive manner. $E M B O$ \% . 8: 1455-1460.

Nellen, W., C. Silan, U. Saur, and R.A. Firtel. 1986. Regulatory sequences in the promoter of the Dictyostelium Actin 6 gene. EMBO I. 5: 3367-3372.

Oyama, M. and D.D. Blumberg. 1986. Interaction of cAMP with the cell surface receptor induces cell-type specific mRNA accumulation in Dictyostelium discoideum. Proc. Natl. Acad. Sci. 83: 4819-4823.

Pavlovic, J., E. Banz, and R.W. Parish. 1986. Hypersensitive sites in the $5^{\prime}$ and $3^{\prime}$ flanking regions of the cysteine proteinase I gene of Dictyostelium discoideum. Nucleic Acids Res. 14: 8703-8722.

Pavlovic, J., B. Haribabu, and R.P. Dottin. 1988. Transmembrane signal transduction regulates gene expression in Dictyostelium discoideum. Dev. Genet. 9: 371-382.

- 1989. Identification of a signal transduction response sequence element necessary for induction of a Dictyoste lium discoideum gene by extracellular cyclic AMP. Mol. Cell. Biol. 9: 4660-4669.

Pears, C.J. and J.G. Williams. 1987. Identification of a DNA sequence element required for efficient expression of a developmentally regulated and cAMP-inducible gene of Dictyostelium discoideum. EMBO J. 6: 195-200.

_ 1988 . Multiple copies of a G-rich element upstream of a cAMP-inducible Dictyostelium gene are necessary but not sufficient for efficient gene expression. Nucleic Acids Res. 16: $8467-8486$.

Pears, C.J., H. Mahbubani, and J.G. Williams. 1985. Characterization of two highly diverged but developmentally co-regulated cysteine proteinase genes in Dictyostelium discoideum. Nucleic Acids Res. 13: 8853-8866.

Pfeifer, K., T. Prezant, and L. Guarente. 1987. Yeast HAPl activator binds to two upstream activation sites of different sequence. Cell 49: 19-27.

Ragheb, J.A. and R.P. Dottin. 1987. Structure and sequence of a UDP glucose pyrophosphorylase gene of Dictyostelium discoideum. Nucleic Acids Res. 15: 3891-3906.

Reymond, C.C., R.H. Gomer, M.C. Mehdy, and R.A. Firtel. 1984. Developmental regulation of a Dictyostelium gene encoding a protein homologous to mammalian ras protein. Cell 39: 141-148.

Schaap, P. and R. Van Driel. 1985. Induction of post-aggregative differentiation in Dictyostelium discoideum by cAMP. Evidence for the involvement of the cell-surface cAMP receptor. Exp. Cell Res. 159: 388-398.

Schaap, P., M. Van Lookeren Campagne, R.V. Van Driel, W. Spek, P.J.M. Van Haastert, and J. Pinas. 1987. Postaggregative differentiation induction by cAMP in Dictyostelium: Intracellular transduction pathway and requirement for additional stimuli. Dev. Biol. 118: 52-63.

Stuart, G.W., P.R. Searle, H.Y. Chen, R.L. Brinster, and R.D. Palmiter. 1984. A 12-base-pair DNA motif that is repeated several times in metallothionein gene promoters confers metal regulation to a heterologous gene. Proc. Natl. Acad. Sci. 81: 7318-7322.

Williams, J.G., A.S. Tsang, and H. Mahbubani. 1980. A change in the rate of transcription of a eukaryotic gene in response to cyclic AMP. Proc. Natl. Acad. Sci. 77: 7171-7175.

Williams, J.G., M.J. North, and H. Mahbubani. 1985. A developmentally regulated cysteine proteinase in Dictyostelium discoideum. EMBO /. 4: 999-1006.

Williams, J.G., A. Ceccarelli, S. McRobbie, H. Mahbubani, R.R. Kay, H. Early, M. Berks, and K.A. Jermyn. 1987. Direct induction of Dictyostelium prestalk gene expression by DIF provides evidence that DIF is a morphogen. Cell 49: 185192. 


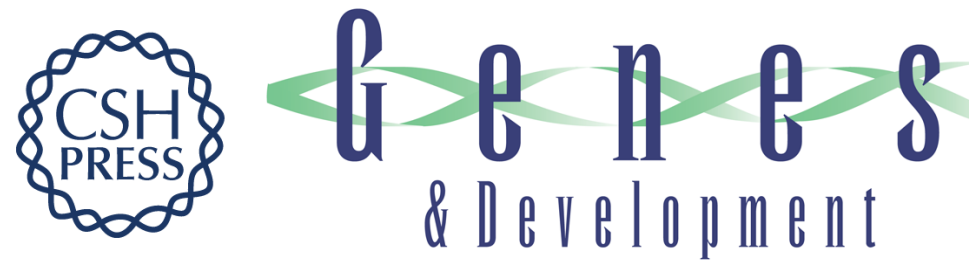

\section{A developmentally regulated trans-acting factor recognizes dissimilar G/C-rich elements controlling a class of cAMP-inducible Dictyostelium genes.}

A L Hjorth, C Pears, J G Williams, et al.

Genes Dev. 1990, 4:

Access the most recent version at doi:10.1101/gad.4.3.419

References This article cites 45 articles, 20 of which can be accessed free at: http://genesdev.cshlp.org/content/4/3/419.full.html\#ref-list-1

License

Email Alerting Receive free email alerts when new articles cite this article - sign up in the box at the top Service right corner of the article or click here.

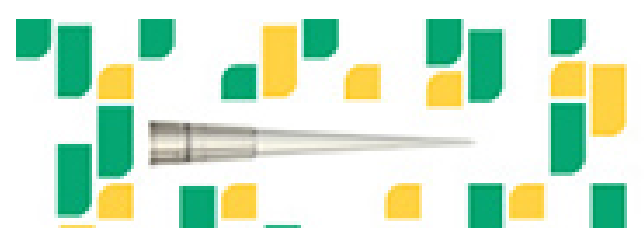

Focused on your science. 